\title{
Release of Aloe vera Extracts from Therapeutic Lenses
}

\author{
Alessandra Pulliero ${ }^{1, *}$, Aldo Profumo ${ }^{2}$, Alberto Izzotti ${ }^{2,3} 3$ and Sergio Claudio Saccà ${ }^{2}$ \\ 1 Department of Health Sciences, University of Genoa, Via A., Pastore 1, 16132 Genoa, Italy \\ 2 IRCCS Ospedale Policlinico San Martino, 16132 Genova, Italy; aldo.profumo@hsanmartino.it (A.P.); \\ izzotti@unige.it (A.I.); sergio.sacca@hsanmartino.it (S.C.S.) \\ 3 Department of Experimental Medicine, University of Genoa, 16132 Genoa, Italy \\ * Correspondence: alessandra.pulliero@unige.it; Tel.: +39-010-353-8509
}

Received: 16 November 2020; Accepted: 15 December 2020; Published: 18 December 2020

\begin{abstract}
Aloe vera extracts have been used for drug therapy due to their antimicrobial, anti-inflammatory, and epithelia-regenerative actions. Considering the possible adverse side effects on the corneal surface of anti-microbial agent administration after ocular surgery, it is of interest to identify the ability of Aloe vera extract release from a therapeutic lens to prevent ocular irritation. The aim of this research was to evaluate the effects of different lens types on the availability and efficacy of releasing Aloe vera extracts in which they are soaked. Two lens types were examined: (1) non-ionic hydrogel lenses and (2) ionic hydrogel lenses. Aloe vera availability was quantified by LC-MS, and activity was quantified by a spectrophotometer (Nanodrop) at different times: (a) $30 \mathrm{~min}$, (b) $60 \mathrm{~min},(\mathrm{c}) 2 \mathrm{~h}$, (d) $6 \mathrm{~h}$, and (e) $24 \mathrm{~h}$. The lens allowing for the most abundant passage of Aloe vera extracts was the ionic hydrogel lens, followed by the non-ionic hydrogel lens. The obtained results demonstrate that the best lens allowing for the high and stable release of Aloe vera extract to the corneal surface are those composed of ionic hydrogels.
\end{abstract}

Keywords: therapeutic lens; Aloe vera extracts; hydrogels; non-ionic lens; ionic lens

\section{Introduction}

Aloe vera extracts have been used for drug therapy due to their antimicrobial, anti-inflammatory, and epithelia-regenerative actions [1]. In the literature, little is known of the ocular use of Aloe vera in therapeutic ocular treatments. Currently, there are no studies considering the interaction between Aloe vera extracts and therapeutic lenses regarding the material composing each lens. Therapeutic lenses provide analgesic and protective effects by shielding epithelial defects and acting as physical bandages for the corneal surface [2]. Ophthalmic drug-delivery systems include the use of poly-(ethylene glycol) hydrogels. Hydrogel lenses with higher water contents adsorb larger amounts of proteins [3]. Hydrogel materials were the most common lens materials for decades, until the recently successful development of truly complex soft lenses (e.g., the silicone hydrogel polymer complex), that possess an affinity to oxygen, improving the oxygen permeability), which account for up to $65 \%$ of today's modern contact lenses. These hydrogels show adequate light transparency, oxygen permeability, and swelling for use as contact lenses [4]. Hydrogel lens may be ionic or non-ionic. Ionic lenses can absorb a thicker layer of protein than non-ionic lenses, the former primarily adsorbing lysozyme [5]. The coverage of the corneal surface reduces the symptoms caused by the exposure of corneal nerve endings. In the last decade, hydrogel use has further expanded due to both the improvement of lens materials and the expansion of therapeutic indications [6]. Hydrophilicity can increase the bioavailability of pharmacological preparations in a solution [7]. The use of therapeutic lenses can alter the physiology of ocular tissue, creating intercellular spaces where foreign particles can be introduced.

However, contact lenses have the potential to create significant ocular complications due to microbial infections. Ocular surface infections are the most feared and significant complication of 
contact lens use. Corneal ulcers are seen with soft contact lens use and abuse [8]. The risk of microbial bacterial keratitis can vary with the type and duration of wear of the lens [9]. Patient factors such as hygiene, smoking, and overnight wear contribute to the overall risk of infections [10]. Aloe vera extracts create a film over the corneal epithelium, thus protecting the eye from these damaging particles, reducing the risk of irritation and infection [11], and protecting from ultraviolet rays [12]. Aloe vera is a natural source of mannose-6-phosphate; this natural-complex sugar can inactivate the action of certain inflammatory mediators via the competitive blocking of keratinocyte receptors from which these mediators exert their action [13]. Aloe vera contains polysaccharides, hydroxy-cinnamic acids, several characteristic anthrones and chromones, the phenolic dimer feralolide, and flavonoids [14]. Of them, the 5-methylchromones aloesin, aloe-emodin, aloenin, aloeresin A, aloesone, and aloin are the active secondary metabolites that have significant beneficial effects in humans. Particularly, aloesin is an aromatic C-glucosylated 5-methylchromone found in Aloe vera that is known to possess anti-oxidant activity [15]. In an in vitro experiment, Ji and Jia [16] found that by incorporating aloin and polysaccharides (found in Aloe vera extracts) into a binary solution, eyes were protected from bacterial infection and UV radiation. This implies that Aloe vera may reduce the toxic effect of irritants, thereby preserving the viability of corneal epithelium cells against the potential long-term toxicity caused by the continuous exposure of the ocular surface.

Considering the possible adverse side effects on the corneal surface of anti-microbial agent administration after ocular surgery, it is of interest to identify the ability of Aloe vera release from therapeutic lens to prevent ocular irritation. Many curative properties of Aloe vera have been reported, including the ability to protect and restore the human corneal epithelium when released from therapeutic lenses [17]. The continuous use of contact lenses may eventually cause irritation and epithelial scratching after surgery. The therapeutic lens may be soaked with Aloe vera to improve beneficial properties and to decrease lipid deposits by reducing the lens' ability to adhere to the corneal surface, thereby improving subjective comfort compared to other lenses.

The goal of the present study was to evaluate the release of Aloe vera extracts in soaked lenses composed of non-ionic or ionic hydrogel commonly used in ocular therapy. The release of Aloe vera by the different types of lenses was evaluated at different times by LC-MS.

\section{Materials and Methods}

\subsection{Therapeutic Lenses}

Two different types of therapeutic lenses (Regenera Therapeutic Lens ${ }^{\circledR}$, Eye Pharma SpA Genoa Italy) were tested with pharmacological eye drops: (1) Contaflex 75\% (C75) (Contamac) non-ionic hydrogel lenses with $75 \%$ water and a refractive index of 1.375 and (2) G72 HW (high water) (Benz Research \& Development) ionic hydrogel lenses with $72 \%$ water and a refractive index of 1.384 . The ionic lens material was Filcon IV 3, a copolymer of 2-hydroxyethylmethacrylate (2-HEMA) and glycerol methacrylate (GMA). The non-ionic lens material was Filcon II 3, a copolymer of methyl methacrylate (MMA) and vinyl polymer (VP). The copolymer consisted of $45 \%$ methafilcon A and $55 \%$ water by weight when immersed in a normal buffered saline solution. The two different type of lens were soaked with Aloe vera extract (Avizor Alvera, Avizor S.A, Madrid, Spain). This Aloe vera extract contained a boric acid buffer, Ethylenediaminetetraacetic acid (EDTA), $0.0002 \%$ polyhexanide, and poloxamer and polyvinylpyrrolidone as surfactant agents.

\subsection{Release of Aloe vera Extract from Therapeutic Lenses}

The release of Aloe vera extract from therapeutic lenses was tested by immersing the lenses in $1 \mathrm{~mL}$ of physiological solution ( $\mathrm{NaCl} 0.9 \%$ ) for $30 \mathrm{~min}, 60 \mathrm{~min}, 2 \mathrm{~h}, 6 \mathrm{~h}$, and $24 \mathrm{~h}$. Each experimental condition was tested in 2 independent replicate experiments for a total of 20 analyses ( 5 experimental conditions $\times 2$ lenses $\times 2$ replicates). The amount of Aloe vera extract passing through lens surface 
was quantified by a UV nano-spectrophotometer (NanoDrop 2000 UV-Vis, Thermofisher Scientific, Wilmington, DE, USA) evaluating absorbance at $230 \mathrm{~nm}$.

\subsection{LC-MS Analysis}

Aloe vera contains more than 75 different compounds, including vitamins (vitamin A, C, E, and B12), enzymes (e.g., amylase a class of enzymes that catalyze the hydrolysis of starch; catalase enzyme that catalyzes the decomposition of hydrogen peroxide to water and oxygen, and peroxidase, enzymes that catalyze the oxidation of a substrate by hydrogen peroxide or an organic peroxide), minerals (e.g., zinc an essential mineral for our body, copper essential to the proper functioning of organs and metabolic processes, selenium an element involved in the protection of cells in the against excess $\mathrm{H}_{2} \mathrm{O}_{2}$, in heavy metal detoxification and calcium), sugars (monosaccharides such as mannose-6-phosphate and polysaccharides such as glucomannans), anthraquinones (aloin and emodin), fatty acids (e.g., lupeol a pentacyclic triterpenoid, in which the hydrogen at the 3beta position is substituted by a hydroxy group and campesterol, a steroid derivative characterized by the hydroxyl group in position C-3 of the steroid skeleton), hormones (auxins and gibberellins), and others (e.g., salicylic acid, a lipophilic monohydroxybenzoic acid, lignin, organic polymers that form key structural materials in the support tissues of vascular plants and saponins a class of bitter-tasting compounds that produce soap-like foam when added to water. Most saponins occur naturally in plants.) $[18,19]$. Among the various water-soluble pharmacological findings compounds for Aloe vera, there is aloesin 2-acetilonil-8-394 142-144, $\beta$-D-glucopraniosyl-7-hydroxy-5-methylchromone, with a molecular weight of 394, that may be used as tracer for a whole mixture [20].

Aloesin release from lenses into the physiological solution was determined by high pressure liquid chromatography conducted using a Vanquish (Thermo Fisher Scientific Wilmington, DE, USA) Ultra High Performance Liquid Chromatography (UHPLC) system composed of a binary pump, an autosampler, and a column oven [21] For aloesin determination, $15 \mu \mathrm{L}$ of each sample were injected onto an Accucore aQ column $(100 \times 2.1 \mathrm{~mm} ; 2.6 \mu \mathrm{m}$ particle size) (Thermo Scientific, San Jose, CA, USA) maintained at $25^{\circ} \mathrm{C}$. The eluents were $0.1 \%$ formic acid (eluent $\mathrm{A}$ ) and acetonitrile (eluent $\mathrm{B}$ ). The flow rate was $200 \mu \mathrm{L} / \mathrm{min}$. The mobile phase was a binary linear gradient in the following sequence: isocratic 30\% B for one min, a linear gradient over the course of $19 \mathrm{~min}$ to $100 \% \mathrm{~B}$, maintained at $100 \% \mathrm{~B}$ for five min, and, finally, a linear gradient to $30 \% \mathrm{~B}$ in two min. The re-equilibration time in $30 \% \mathrm{~B}$ was six min. After HPLC separation, the eluent was directly sent to a Q Exactive Plus Orbitrap mass spectrometer (Thermo Scientific, San Jose, CA, USA) equipped with a heated electrospray ionization source (HESI-II). Before the start of each acquisition series, the mass spectrometer was externally calibrated with a negative ion calibration solution (Thermo Fisher Scientific, San Jose, CA, USA). Negative full-scan mass spectra were recorded in the mass range of $100-700 \mathrm{~m} / \mathrm{z}$. The following operation parameters were applied: the sheath and auxiliary gas flow rate were 45 and 10, respectively; the spray voltage was $3.5 \mathrm{kV}$, the capillary temperature was $40{ }^{\circ} \mathrm{C}$; the S-lens (radio frequency) RF level was 100 . The software used for operating the ultra-high-pressure liquid chromatography with high resolution/high accuracy Orbitrap ${ }^{\circledR}$ mass spectrometry (UHPLC/HR-MS) (Thermo Fisher Scientific, San Jose, CA, USA) was Xcalibur (version 4.1). The full scan data were processed, and the amount of aloesin was measured via the extracted ion current (EIC) peak area $(m / z 393.11$ [M-H]-).

\subsection{Statistical Analysis}

Differences between therapeutic lenses were determined using the non-parametric Kruskal Wallis test. A threshold $p$ value of $<0.05$ was considered as statistically significant. Results are presented as mean \pm standard error of the mean (SEM). 


\section{Results}

\subsection{Time Course and Aloe vera Release from the Therapeutic Lenses}

Figure 1 reports the construction of the standard reference curve for Aloe vera extracts obtained by diluting the extract to $1 / 1,1 / 10,1 / 50,1 / 100$, and $1 / 200$.

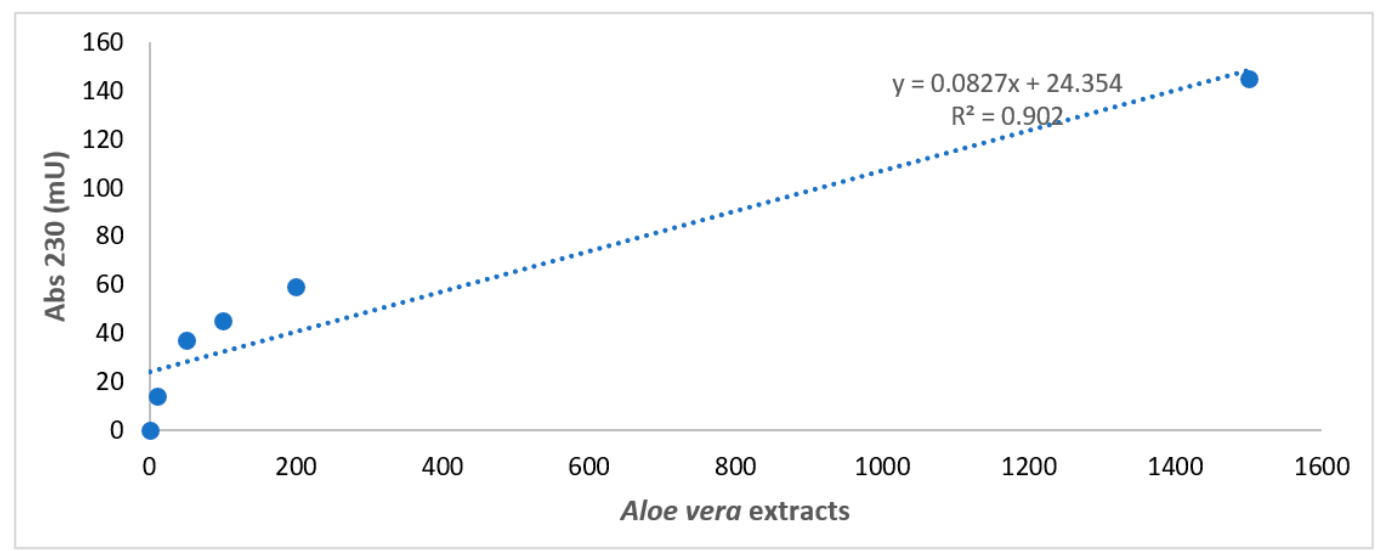

Figure 1. Spectrophotometer analysis of Aloe vera extracts. Spectrophotometer analysis of Aloe vera extracts obtained by diluting the extract to $1 / 1,1 / 10,1 / 50,1 / 100$, and $1 / 200$ in a physiologic solution with a standard reference curve. $X$ axis: wavelength in $\mathrm{nm}$. $\mathrm{Y}$ axis: absorbance intensity at $230 \mathrm{~nm}$.

The time course releases of Aloe vera extract from lenses, as evaluated by UV nano-spectrophotometry, are reported in Figure 2.

(a)

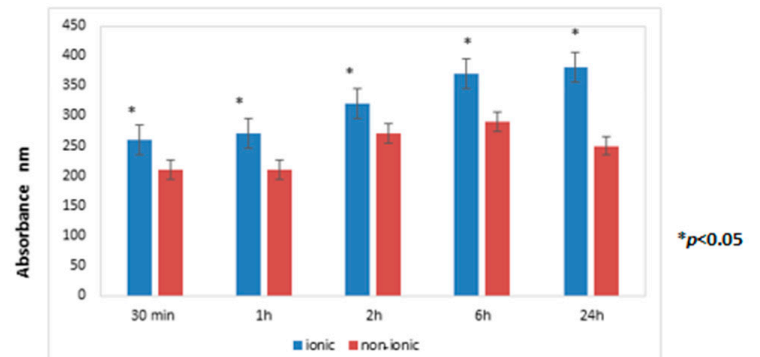

(b)
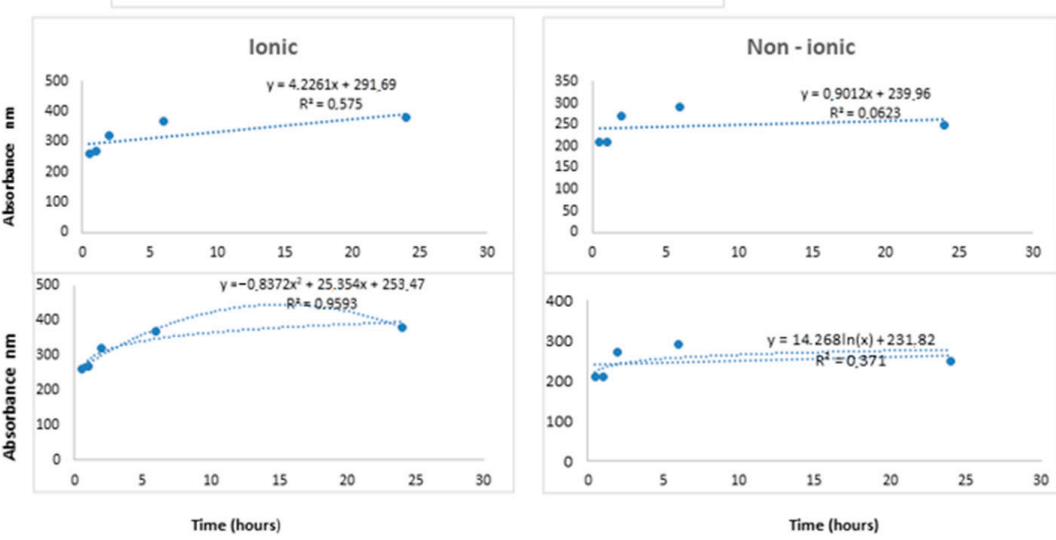

Figure 2. (a) The time course releases of Aloe vera extract from lenses. At all times tested ( $30 \mathrm{~min}, 1 \mathrm{~h}$, $2 \mathrm{~h}, 6 \mathrm{~h}$, and $24 \mathrm{~h}$ ), the lens allowing for the most abundant passage of Aloe vera was the ionic hydrogel lens (G72 HW (high water) lens) as compared to the non-ionic hydrogel lens (C75). (b) The linear regression analysis in the upper panel and the log regression equation model is represented in the lower panel. These differences were statistically significant $\left.{ }^{*} p<0.05\right)$. 
At all times tested ( $30 \mathrm{~min}, 1 \mathrm{~h}, 2 \mathrm{~h}, 6 \mathrm{~h}$, and $24 \mathrm{~h}$ ), the lens allowing for the most abundant passage of Aloe vera was the ionic hydrogel lens (G72 HW (high water) lens) compared to the non-ionic hydrogel lens (C75). These differences were statistically significant $(p<0.05)$.

The kinetic of Aloe vera release was comparatively evaluated for the two lenses by performing linear regression analysis. For the ionic lens, the regression line equation displayed a highly positive angular coefficient (4.2261) with a high $R_{2}$ value (0.5750), thus indicating a remarkable relation between the two analyzed variables, i.e., time and Aloe vera extract release. This finding indicated that the release of the extract from the ionic lens steadily persisted at all tested times.

For the non-ionic lens, the regression line equation displayed a barely positive angular coefficient (0.9012) with a low $\mathrm{R}_{2}$ value (0.0623), thus indicating a poor relationship between the two analyzed variables, i.e., time and Aloe vera extract release. This finding indicated that the release of extract from the non-ionic lens was minimal and did not persist at all tested times.

\subsection{LC-MS Analysis}

An LC-MS analysis successfully detected the aloesin content in the aqueous media after the release of therapeutic lens incubation, as seen in Figure 3.

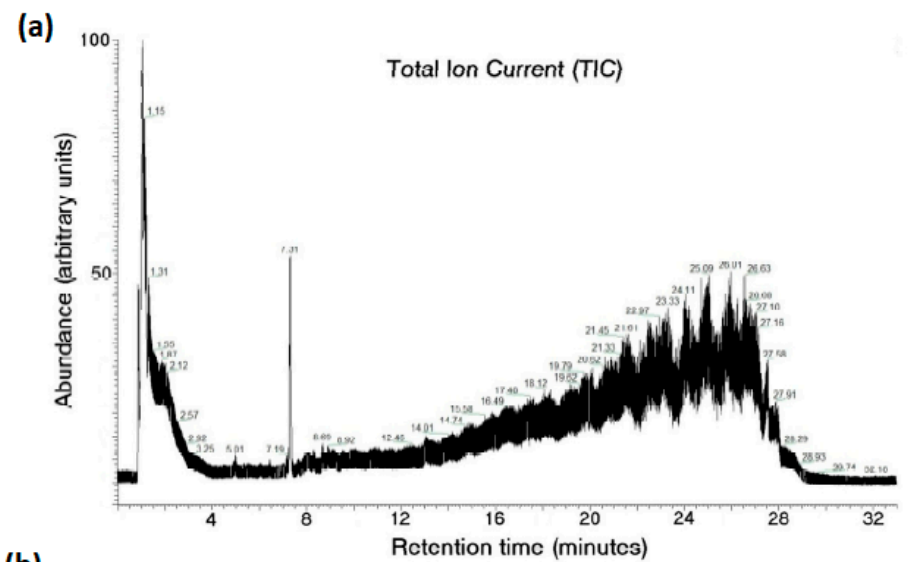

(b)

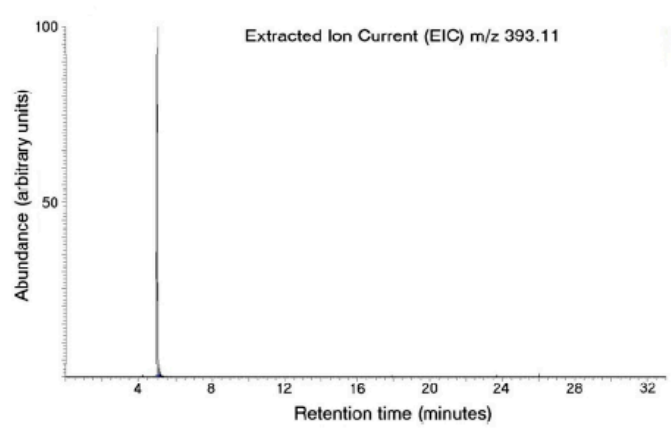

(c)

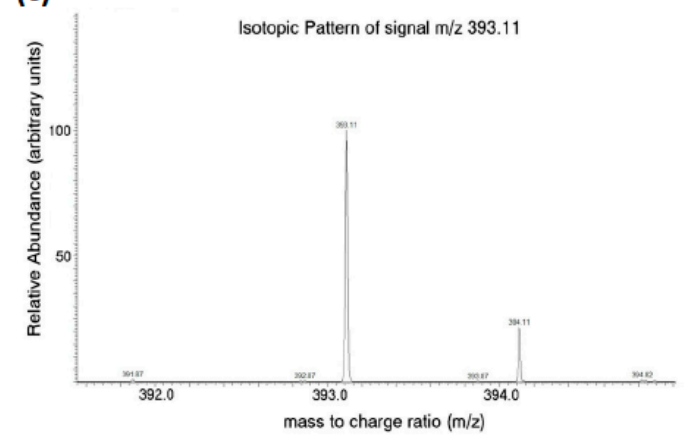

Figure 3. Aloesin content in the aqueous media after the release of therapeutic lens incubation measured with LC-MS analysis. The total ion current from the analysis revealed the complexity of the examined mixture (a). Aloesin was identified by the extracted ion current (b) and isotopic pattern analysis (c) using an $m / z$ of 393.11 .

The total ion current from the analysis revealed the complexity of the examined mixture (Figure 3a). In this context, aloesin was identified by extracted ion current (Figure $3 b$ ) and isotopic pattern analysis (Figure 3c) using an $m / z$ of 393.11 .

The amount of aloesin released from the two lenses was different, as demonstrated by the overlapping peaks reported in Figure 4. 

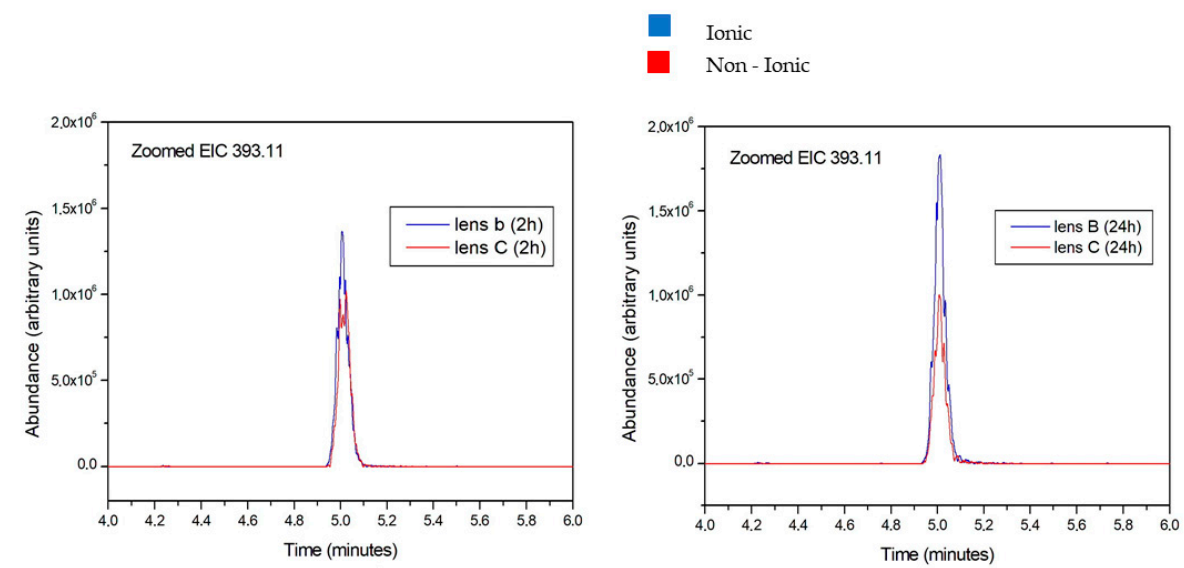

Figure 4. Aloesin amount released from ionic and non-ionic lens after 2 and $24 \mathrm{~h}$. The blue peak overwhelms the red peak at all tested times, thus indicating the more abundant release of aloesin from the ionic lens compared to the non-ionic lens.

The aloesin amounts released from ionic and non-ionic lens are reported in blue and in red colors, respectively. Figure 4 reports data obtained at 2 and $24 \mathrm{~h}$. The blue peak overwhelms the red peak at all tested times, thus indicating the more abundant release of aloesin from the ionic lens compared to the non-ionic lens.

Furthermore, the blue peak is higher at $24 \mathrm{~h}$ than at $2 \mathrm{~h}$, indicating an increasing release of aloesin from the ionic lens over time. Conversely, the red peak is lower at $24 \mathrm{~h}$ than at $2 \mathrm{~h}$, indicating a decreased release of aloesin from the non-ionic lens over time.

The quantification of these results for all tested times is reported in Figure 5.
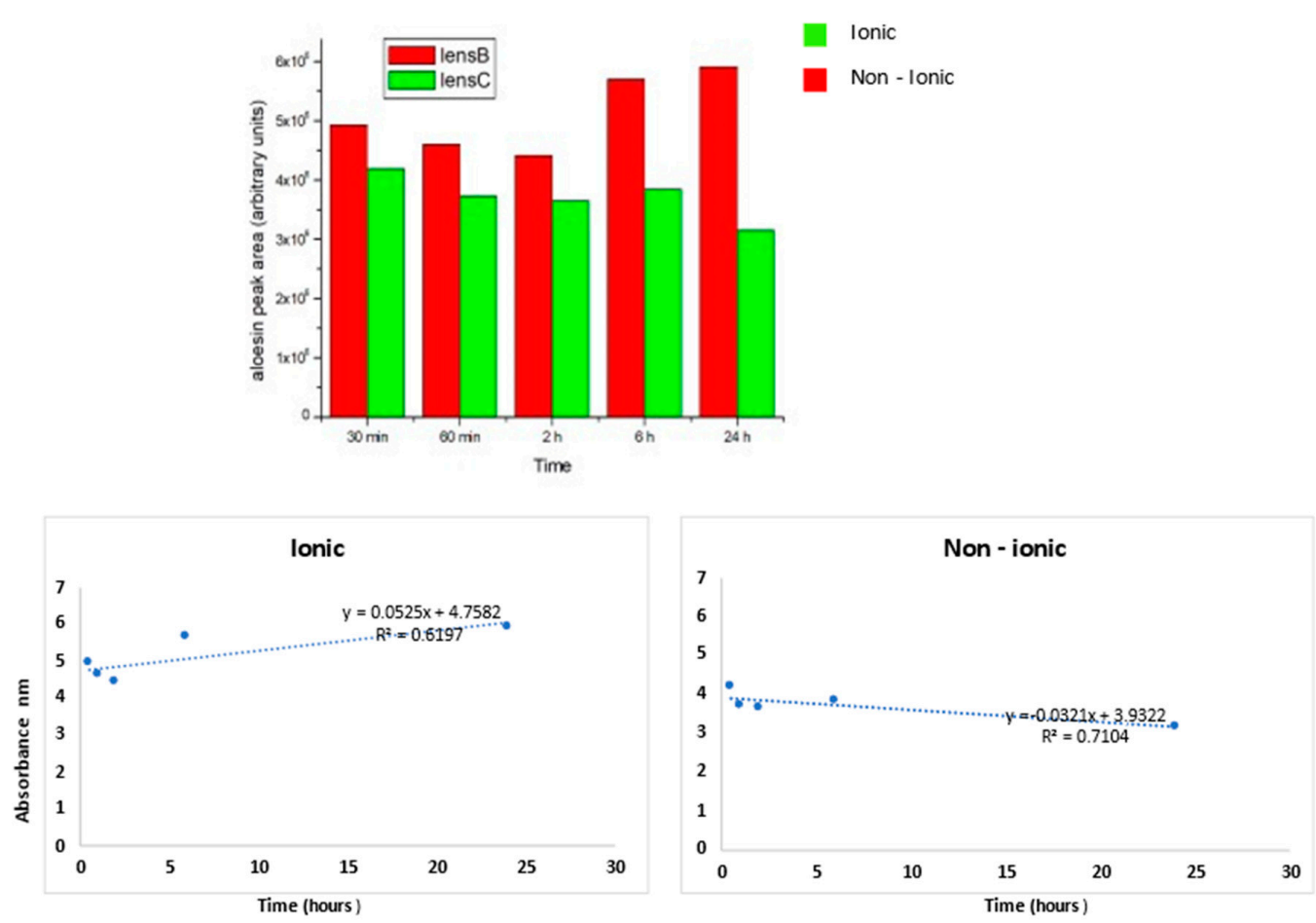

Figure 5. The aloesin release was higher for the ionic lens than non-ionic lens at all times tested. For the ionic lens, the regression line equation displays a positive angular coefficient with a high $\mathrm{R}_{2}$ value, thus indicating a continuous aloesin release from the lens increasing from 2 to $24 \mathrm{~h}$. For the non-ionic lens, the regression line equation displays a negative angular coefficient with a high $\mathrm{R}_{2}$ value, thus indicating a decrease of aloesin release from the lens from 2 to $24 \mathrm{~h}$. 
As inferred from the histogram, the aloesin release was higher for the ionic lens than for the non-ionic lens at all times tested. For the ionic lens, the regression line equation displays a positive angular coefficient with a high $R_{2}$ value, thus indicating a continuous aloesin release from the lens increasing from 2 to $24 \mathrm{~h}$. For the non-ionic lens, the regression line equation displays a negative angular coefficient with a high $R_{2}$ value, thus indicating a decrease of aloesin release from the lens from 2 to $24 \mathrm{~h}$.

The release kinetics for the ionic lens underwent two phases: the early phase $(0.5-2 \mathrm{~h})$ characterized by a slow release and a late phase $(6-24 \mathrm{~h})$ characterized by a high and increasing release. These kinetics were not observed for non-ionic lens, where a stable decrease over time of the release was observed.

\section{Discussion}

The obtained results indicated that release of Aloe vera extract from the lens was different in the ionic and non-ionic lenses. These findings bear clinical relevance because therapeutic contact lenses are an option to treat ocular diseases due to their ability to release drugs for extended periods of time. Therefore, the critical properties of lenses, such as water content and ion and oxygen permeability for comfortable day and night wear contact, are important topics of research to enable their therapeutic uses. We evaluated the release of Aloe vera extract for two different types of therapeutic lenses. The non-ionic hydrogel lens that was tested, with $75 \%$ water, is a type of lens in which the water content is suited to bandaging the corneal surface. The hydration of these materials is best performed in buffered saline with a $\mathrm{pH}$ of 6.8-7.3. The tested ionic hydrogel lens had a $72 \%$ water content and met the criteria for high-performance daily-wear lenses. We analyzed the release of Aloe vera extracts at five different times, comparing ionic and non-ionic therapeutic lenses. In our experiment, we verified that the ionic hydrogel lens released a higher quantity of aloesin than the non-ionic hydrogel lens for all the analysis times considered (after $30 \mathrm{~min}, 60 \mathrm{~min}, 2 \mathrm{~h}, 6 \mathrm{~h}$, and $24 \mathrm{~h}$ ). Hydrogels have proven to be an extremely versatile class of materials with many potential applications in ophthalmology. A hydrogel material is porous and traps aloesin.

Our hypothesis is that aloesin remained passively trapped in the non-ionic lens, and the release was therefore lesser and decreased over time. Conversely, in the ionic lens, the negative charge rejected the aloesin, thus making its release continuous possible and leading to a steadily increasing release to the outside in the medium. Therefore, the release was greater and more prolonged over time for the ionic lens compared to the non-ionic lens. However, there was no measure of how much aloesin was absorbed by each lens, so we do not know how much was remaining in each lens (although it was clear that the ionic lens released more). Ionic hydrogel lenses are high performance soft lens materials because they are able to stay completely hydrated and dimensionally stable, as well as extremely wettable, on the eye. Staying hydrated during wear means that a $54 \%$ water, high performance G-(Glycerol Methacrylate) material lens can provide an oxygen transmission of $20 \mathrm{Dk} / \mathrm{T}$ at an average lens thickness of 105 microns, and a $75 \%$ water content G-material lens can provide $20 \mathrm{Dk} / \mathrm{T}$ all the way to an average lens thickness 300 microns. This means that virtually any lens design can be a high performance daily wear lens. These high performance lens properties are a function of the polymer's water compatibility [22]. For material comparison, it is useful to examine the percent change in the pure saline contact angle between each material rather than a particular angle. The contact angle is reduced by $24 \%$ in going from a poly-HEMA-based lens to a $54 \%$ GMA/HEMA copolymer lens. This amount of change in contact angle may be what is necessary for patients to consistently have a comfort preference between two materials. Thus, the "spikiness" of lenses may also influence adhesion by reducing adhesion to both hydrophilic and hydrophobic lenses. In turn, this effect could be especially relevant for hydrophilic lenses [23].

The results of the present study highlight the relevance of how the lens material is fundamental in conditioning the release of drugs from therapeutic lenses with reference to extracts of Aloe vera. The hydrogel non-ionic material had a lower water content than the ionic material. The amount of drug a contact lens can absorb, as well as the drug's release, is influenced by the lens' water content [24]. 
The dehydration of conventional hydrogel lenses significantly impacts the oxygen permeability or the transmissibility value of the lens [24-27]. The long-term consequences of changes induced by lens-dehydration include corneal hypoxia, corneal vascularization, a decreased transparency, compromised endothelial function, and an increased susceptibility to infections. The water layer of the tear film that covers the anterior surface of the contact lens when placed over the cornea can be lost for several reasons, diminishing the water content of the hydrogel lens, thus causing a decrease in the amount of oxygen reaching the corneal surface. This situation can be responsible for significant corneal staining, dehydration, ocular dryness, and neovascularization [28].

Aloe vera contains multiple pharmacologically active substances that are capable of modulating cellular metabolism, activating protective enzymes, and activating antibacterial, antifungal, and anti-inflammatory activities [29]. It should be noted that the source material for existing Aloe vera extracts may undergo a remarkable variation between different studies and products. Indeed, there is no consistent and standardized extraction method, nor is there an established quality criterion for selecting the plants that are the natural source of these extracts. Accordingly, the evaluation of aloesin concentration that we tested in the presented study may be proposed as a reference standard to analyze Aloe vera extract quality and pharmaco-dynamics.

Though the time course of the Aloe vera extract release from ionic vs. non-ionic lens was statistically significant, differences were small. Accordingly, whether or not the two lenses behave similarly should be further examined in future clinical studies.

\section{Conclusions}

In conclusion, the herein-presented results provide evidence that the use of Aloe vera extracts for ocular therapeutic or preventive purposes is a complex situation where many variables should be considered. These variables include (a) the quality of Aloe vera extract indicated by the amount of aloesin content; (b) the interaction between Aloe vera extract and lens material, a situation remarkably affecting the pharmaco-dynamic properties of Aloe vera extracts; and (c) the composition of hydrogel material composing the lens in terms of ionic or non-ionic materials. The obtained results demonstrated that the best lenses allowing for the high and stable release of Aloe vera extract to the corneal surface are those composed of ionic hydrogels.

Author Contributions: Conceptualization, A.I. and S.C.S.; methodology, A.P. (Aldo Profumo); software, A.P. (Aldo Profumo); validation, A.P. (Alessandra Pulliero), A.P. (Aldo Profumo) and S.C.S.; formal analysis, A.P. (Alessandra Pulliero); investigation, A.I.; resources, A.I.; data curation, A.P. (Alessandra Pulliero); writing —original draft preparation, A.P. (Alessandra Pulliero); writing—review and editing, A.I.; visualization, S.C.S.; supervision, A.I.; project administration, A.I.; funding acquisition, A.I. All authors have read and agreed to the published version of the manuscript.

Funding: This research did not receive external funding.

Conflicts of Interest: The authors declare no conflict of interest.

\section{References}

1. Rahmani, A.H.; Aldebasi, Y.H.; Srikar, S.; Khan, A.A.; Aly, S.M. Aloe vera: Potential candidate in health management via modulation of biological activities. Pharmacogn. Rev. 2015, 9, 120-126. [CrossRef] [PubMed]

2. Shah, C.; Raj, C.; Foulks, G.N. The evolution in therapeutic contact lenses. Ophthalmol. Clin. N. Am. 2003, 16, 95-101. [CrossRef]

3. Omali, N.B.; Subbaraman, L.N.; Coles-Brennan, C.; Fadli, Z.; Jones, L.W. Biological and Clinical Implications of Lysozyme Deposition on Soft Contact Lenses. Optom. Vis. Sci. 2015, 92, 750-757. [CrossRef] [PubMed]

4. Alvarez-Rivera, F.; Rey-Rico, A.; Venkatesan, J.K.; Diaz-Gomez, L.; Cucchiarini, M.; Concheiro, A.; Alvarez-Lorenzo, C. Controlled Release of rAAV Vectors from APMA-Functionalized Contact Lenses for Corneal Gene Therapy. Pharmaceutics 2020, 12, 335. [CrossRef] 
5. Teichroeb, J.H.; Forrest, J.A.; Ngai, V.; Martin, J.W.; Jones, L.; Medley, J. Imaging Protein Deposits on Contact Lens Materials. Optom. Vis. Sci. 2008, 85, 1151-1164. [CrossRef]

6. Fonn, D.; E MacDonald, K.; Richter, R.; Pritchard, N. The ocular response to extended wear of a high Dk silicone hydrogel contact lens. Clin. Exp. Optom. 2002, 85, 176-182. [CrossRef]

7. Başaran, E.; Yazan, Y. Ocular application of chitosan. Expert Opin. Drug Deliv. 2012, 9, 701-712. [CrossRef]

8. Lim, C.H.L.; Stapleton, F.; Mehta, J.S. Review of Contact Lens-Related Complications. Eye Contact Lens: Sci. Clin. 2018, 44, S1-S10. [CrossRef]

9. Cheng, K.H.; Leung, S.L.; Hoekman, H.W.; Beekhuis, W.H.; Mulder, P.G.; Geerards, A.J.; Kijlstra, A. Incidence of contact-lens-associated microbial keratitis and its related morbidity. Lancet 1999, 354, 181-185. [CrossRef]

10. Liesegang, T.J. Contact lens-related microbial keratitis: Part I: Epidemiology. Cornea 1997, 16, $125-131$. [CrossRef]

11. Vecchione, A.; Celandroni, F.; Lupetti, A.; Favuzza, E.; Mencucci, R.; Ghelardi, E. Antimicrobial Activity of a New Aloe vera Formulation for the Hygiene of the Periocular Area. J. Ocul. Pharmacol. Ther. 2018, 34, 579-583. [CrossRef] [PubMed]

12. Misawa, E.; Tanaka, M.; Saito, M.; Nabeshima, K.; Yao, R.; Yamauchi, K.; Abe, F.; Yamamoto, Y.; Furukawa, F. Protective effects of Aloe sterols against UVB-induced photoaging in hairless mice. Photodermatol. Photoimmunol. Photomed. 2017, 33, 101-111. [CrossRef] [PubMed]

13. Angunawela, R.I.; Marshall, J. Inhibition of transforming growthfactor-beta1 and its effects on human corneal fibroblastsby mannose-6-phosphate: potential for preventing hazeafter refractive surgery. J. Cataract. Refract. Surg. 2010, 36, 121-126. [CrossRef] [PubMed]

14. Loots, D.T.; Van Der Westhuizen, F.H.; Botes, L. Aloe feroxLeaf Gel Phytochemical Content, Antioxidant Capacity, and Possible Health Benefits. J. Agric. Food Chem. 2007, 55, 6891-6896. [CrossRef]

15. Lucini, L.; Pellizzoni, M.; Pellegrino, R.; Molinari, G.P.; Colla, G. Phytochemical constituents and in vitro radical scavenging activity of different Aloe species. Food Chem. 2015, 170, 501-507. [CrossRef]

16. Jia, Y.; Jia, J. Ultraviolet Light Absorption of an Ophthalmic Formulation with Aloe Extracts. Nat. Prod. Commun. 2009, 4, 1231-1233. [CrossRef]

17. Woźniak, A.; Paduch, R. Aloe vera extract activity on human corneal cells. Pharm. Biol. 2012, 50, 147-154. [CrossRef]

18. Malik, I. Aloe vera-A Review of its Clinical Effectiveness. Int. Res. J. Pharm. 2013, 4, 75-79. [CrossRef]

19. Maan, A.A.; Nazir, A.; Khan, M.K.I.; Ahmad, T.; Zia, R.; Murid, M.; Abrar, M. The therapeutic properties and applications of Aloe vera: A review. J. Herb. Med. 2018, 12, 1-10. [CrossRef]

20. Sánchez, M.; González-Burgos, E.; Iglesias, I.; Gómez-Serranillos, P.M. Pharmacological Update Properties of Aloe vera and its Major Active Constituents. Molecules 2020, 25, 1324.

21. Wahedi, H.M.; Jeong, M.; Chae, J.K.; Gil Do, S.; Yoon, H.; Do, S.-G. Aloesin from Aloe vera accelerates skin wound healing by modulating MAPK/Rho and Smad signaling pathways in vitro and in vivo. Phytomedicine 2017, 28, 19-26. [CrossRef] [PubMed]

22. Guryca, V.; Hobzova, R.; Pradny, M.; Sirc, J.; Michalek, J. Surface mor-phology of contact lenses probed with microscopy techniques. Cont. Lens Anterior Eye 2007, 30, 215-222. [CrossRef] [PubMed]

23. Giráldez-Fernández, M.; Resua, C.G.; Lira, M.; Oliveira, M.E.C.D.R.; Magariños, B.; Toranzo, A.E.; Yebra-Pimentel, E. Contact Lens Hydrophobicity and Roughness Effects on Bacterial Adhesion. Optom. Vis. Sci. 2010, 87, E426-E431. [CrossRef]

24. Ramamoorthy, P.; Sinnott, L.T.; Nichols, K.K. Contact lens material characteristics associated with hydrogel lens dehydration. Ophthalmic Physiol. Opt. 2010, 30, 160-166. [CrossRef]

25. Grzech, A.; Misiuk-Hojło, M. The meaning of oxygen permeability in different materials for optimalization of contact lenses function. Polym. Med. 2007, 37, 67-71.

26. Gu, X.-J.; Liu, X.; Chen, Y.-Y.; Zhao, Y.; Xu, M.; Han, X.-J.; Liu, Q.-P.; Yi, J.-L.; Li, J.-M. Involvement of NADPH oxidases in alkali burn-induced corneal injury. Int. J. Mol. Med. 2016, 38, 75-82. [CrossRef]

27. Efron, N.; Morgan, P.B. Hydrogel contact lens dehydration and oxygen transmissibility. CLAO J. 1999, $25,148-151$.

28. Chigbu, D.G.I. The pathophysiology of ocular allergy: A review. Contact Lens Anterior Eye 2009, 32, 3-15. [CrossRef] 
29. Williams, M.S.; Burk, M.; Loprinzi, C.L.; Hill, M.; Schomberg, P.J.; Nearhood, K.; O’Fallon, J.R.; Laurie, J.A.; Shanahan, T.G.; Moore, R.L.; et al. Phase III double-blind evaluation of an Aloe vera gel as a prophylactic agent for radiation-induced skin toxicity. Int. J. Radiat. Oncol. Biol. Phys. 1996, 36, 345-349. [CrossRef]

Publisher's Note: MDPI stays neutral with regard to jurisdictional claims in published maps and institutional affiliations.

(C) 2020 by the authors. Licensee MDPI, Basel, Switzerland. This article is an open access article distributed under the terms and conditions of the Creative Commons Attribution (CC BY) license (http://creativecommons.org/licenses/by/4.0/). 